\title{
Sex accelerates adaptation
}

\section{A study confirms the classic theory that sex increases the rate of adaptive evolution by accelerating the speed at which beneficial mutations sweep through populations. See Letter p.XXX}

\section{Matthew R. Goddard}

Compared to the asexual alternative of simple cloning, sex seems like a complicated way of reproducing. Fast and efficient reproduction is at the heart of Darwinian natural selection, so why sex exists is a conundrum that has fascinated biologists for more than 100 years $^{1}$. In a paper online in Nature, McDonald et $a .^{2}$ directly confirm the long-held theory that the advantage of sex lies in its ability to expose individual mutations to the actions of natural selection.

Sex involves the shuffling (recombination) of chromosomes from different parents, followed by the separation of these newly-shuffled chromosomes into reproductive gametes, which then fuse through mating. As well as being more complicated than asexual reproduction, sex also risks breaking apart collections of genes that have proven effective. In animals, sex means that fewer offspring are produced as only females give birth, and mate finding and courtship impose further uncertainties. Given these disadvantages, it is not immediately clear why sexual reproduction is maintained.

Mutations accrue in organisms' genomes over time, and some affect ability to reproduce and compete for resources (fitness). The net fitness of an individual is the sum of these various accrued mutations. In asexually reproducing populations classic theories ${ }^{3,4}$ suggest selection only ever 'sees' this net genomic fitness value. When a positive mutation arises in a genome already harbouring negative ones, these might overwhelm the positive mutation, and the whole genome would be removed from the population by natural selection. The 
positive mutation is lost. However, if the positive mutation confers a strong enough fitness benefit to outweigh the negative ones, the genome will likely increase in abundance over generations, and possibly become fixed in the population due to positive selection. Here negative mutations become common by hitchhiking with positive ones, and thus restrict the overall fitness of the population as they do. In asexual populations individual mutations may be masked from the actions of selection as they are entangled in genomes.

Sexual populations do not theoretically suffer this problem ${ }^{3,4}$. Recombination and the random partitioning of chromosomes allow positive mutations to be isolated and disassociated from negative ones. By analogy, sex allows selection to pluck rubies from rubbish ${ }^{5}$. Furthermore, since positive mutations usually arise in different genomes, sex enables these to be recombined into the same genome rather than competing against one another, as they would in an asexual population 4 . In sexual populations, many positive mutations that are mostly free of mutational rubbish can become common simultaneously, and this is predicted to increase the rate and extent of adaptive evolution ${ }^{1}$.

A series of experimental evolution studies support the idea that sex speeds adaptive evolution ${ }^{6-8}$. However, much less work has focused on the molecular mechanisms underpinning this advantage. One study ${ }^{9}$ inferred sex accelerated adaptation by isolating positive mutations from negative ones, but did not directly show the kinds of mutation that arose in the experiment.

McDonald et al., however, have done just that. First, they evolved sexual and asexual yeast (Saccharomyces cerevisiae) populations for approximately 1000 generations in a simple laboratory environment, to which the sexual populations adapted more rapidly. Then, advancing on the work of previous studies, the authors used powerful DNA sequencing approaches to dissect and track various single-base mutations that arose by evaluating populations at regular time points during evolution. 
Initially a similar range of mutations that did and did not affect protein function arose in all populations, and the study reasonably assumed only those that changed function affected fitness. However, a different spectrum of mutations eventually became common in sexual and asexual populations. In asexual populations, different types of mutations were correlated and all had roughly the same chance of becoming fixed, indicating selection could not discriminate between individual mutations. However, by contrast, fewer mutations became fixed in sexual populations, and those that did mostly altered protein function, and thus also presumably fitness. This observation suggests sex improved the efficiency with which selection acted on individual mutations.

To directly test the fitness effects of specific mutations McDonald et al. observed, they conducted controlled separate mini-evolution experiments and tracked the change in frequency of these mutations. This key step allowed McDonald et al. to show groups of positive and negative mutations remained together in asexual populations. These mutation groups (genomes) competed against one another: some became common over generations meaning negative mutations persisted by hitchhiking. By contrast, no mutation groups persisted in sexual populations due to recombination, and negative mutations did not become common. This shows selection more effectively discriminated between individual mutations and eliminated negative while favouring positive ones.

These comprehensive experiments provide the long-awaited confirmation that sex accelerates adaptation by plucking mutational rubies from the rubbish. Sex shuffles mutations among genomes, enabling natural selection to act on individual mutations more efficiently. Selection is comparatively blinded in asexual populations, as the effects of individual mutations are consistently hidden in genomes.

Several aspects of sexual reproduction still remain incompletely understood. First, McDonald and colleagues' study mainly examined only changes in single DNA bases. However, mutations that duplicate, remove or rearrange whole 
segments of DNA are also important for adaptation. As the authors' acknowledge, the effect of sex on these mutations remains to be evaluated.

Second, this study used yeast with one copy of every chromosome, but most sexual species have two, including humans, and natural selection works slightly differently when there are two chromosomes. Third, most species inhabit complex environments that have a variety of selection pressures whose strength varies over space and time. While the current study elegantly shows how sex provides advantages during adaptation to simple environments, it is not clear how this translates to more complex environments. Some work suggests that sex can also accelerate adaptation to complex environments ${ }^{10}$; however, the underlying molecular mechanisms are not known.

Finally, we do not yet know why sex arose in the first place. One theory suggests parasitic genetic elements, which persist in genomes despite conferring no fitness benefit, might promote cell fusion and recombination ${ }^{11}$. However, few experiments have tested this theory ${ }^{12}$. It might well be that the evolution of sex was driven by completely different forces to those, neatly defined by McDonald et al., that we now know to maintain it.

Matthew R Goddard is at The School of Life Sciences, University of Lincoln, Lincoln LN6 7TS, UK, and at The School of Biological Sciences, The University of Auckland, Auckland 1142, New Zealand.

e-mail: mgoddard@lincoln.ac.uk

1. Burt, A. Perspective: Sex, recombination, and the efficacy of selection - Was Weismann right? Evolution 54, 337-351 (2000).

2. McDonald, M., Desai, M. M. \& Rice, D. P. Nature XXX (2016).

3. Fisher, R. A. The Genetical Theory of Natural Selection. (Oxford University Press, 1930).

4. Muller, H. J. Some genetic aspects of sex. Am Nat 66, 118-138 (1932).

5. Peck, J. R. A ruby in the rubbish: beneficial mutations, deleterious 
mutations and the evolution of sex. Genetics 137, 597-606 (1994).

6. Colegrave, N. Sex releases the speed limit on evolution. Nature 420, 664666 (2002).

7. Goddard, M. R., Godfray, H. C. J. \& Burt, A. Sex increases the efficacy of natural selection in experimental yeast populations. Nature 434, 636-640 (2005).

8. Morran, L. T., Schmidt, O. G., Gelarden, I. A., Parrish, R. C. \& Lively, C. M. Running with the Red Queen: host-parasite coevolution selects for biparental sex. Science 333, 216-218 (2011).

9. Gray, J. C. \& Goddard, M. R. Sex enhances adaptation by unlinking beneficial from detrimental mutations in experimental yeast populations. BMC Evol Biol 12, 43 (2012).

10. Gray, J. C. \& Goddard, M. R. Gene-flow between niches facilitates local adaptation in sexual populations. Ecology Letters 15, 955-962 (2012).

11. Hickey, D. A. \& Rose, M. R. in The Evolution of Sex: An Examination of Current Ideas (eds. Michod, R. E. \& Levin, B. R.) 161-193 (Sinauer, 1988).

12. Giraldo-Perez, P. \& Goddard, M. R. A parasitic selfish gene that affects host promiscuity. Proceedings of the Royal Society B: Biological Sciences 280, 20131875-20131875 (2013).

Figure 1 | Picking rubies from the rubbish. Over time, genomes accrue mutations that either benefit (green) or harm (red) an individual's fitness (in this simple schematic, the relative benefit or cost of each mutation is indicated by size). McDonald et al. ${ }^{1}$ compared how asexual and sexual populations allow selection to act on mutations. a, During asexual reproduction, selection occurs based on overall genome fitness. As such, positive mutations may be removed from the population, and negative mutations can hitchhike along with a positive one of greater value. b, During sexual reproduction, chromosomes are shuffled by recombination, changing the mutations that are grouped together in offspring. This process enables individual mutations to be independently retained or removed by selection. 